\section{Section Editor}

Mitchell S.V. Elkind,

MD, MS

\title{
Clinical Reasoning: Unusual headaches
}

Maria Joana Osorio, MD SECTION 1

Poonam Bhatia, MD

Giulio Zuccoli, MD

Deborah Holder, MD

Address correspondence and reprint requests to Dr. Maria Joana Osorio, Division of Child

Neurology, Department of Pediatrics, Children's Hospital of Pittsburgh of UPMC, One Children's Hospital Drive, 4401 Penn Avenue, Pittsburgh, PA 15224

joana.osorio@chp.edu osoriomj@gmail.com
A 14-year-old girl presented to the neurology clinic complaining of headaches. She described her headaches as mild (intensity 3/10), holocranial, and pounding in character. There was no nausea or vomiting, or photophobia or phonophobia. The patient described in detail a sequence of symptoms from the onset of her headaches. First she experienced "funny vision changes in her right eye" characterized by a very bright spinning colored wheel and scotomas. These visual experiences gave rise to right leg numbness, lasting a total of 45 seconds. Following the leg numbness, only the headache would persist, for no longer than $5 \mathrm{~min}$ utes. The patient reported no change in consciousness during these symptoms.
These episodes started 6 months prior to the patient's presentation at the neurology clinic and had progressed with increased frequency from monthly to weekly. In between the episodes, she had no complaints. There was no history of head trauma and the patient's past medical history was unremarkable. Family history was only significant for a maternal cousin with migraines. The patient's general and neurologic examination findings were normal. She was asymptomatic during the examination.

\section{Questions for consideration:}

1. What other questions are pertinent for this case?

2. Where would you localize the symptoms?

\section{SECTION 2}

We compiled a detailed description of the patient's visual complaints in an attempt to localize her symptoms. Additional questioning revealed that when she closed the right eye she still had visual symptoms in the nasal field of the left eye. Although a right monocular field involvement was initially suspected, these additional details instead suggested a right homonymous hemianopia. The patient's initial description of the headache as holocranial suggested a generalized process; however, the right visual field symptoms and the right leg numbness suggested localization to the left occipital and parietal cortex, respectively.

\section{Questions for consideration:}

1. What differential diagnosis would you consider at this time?

2. What diagnostic tests should be ordered at this time?

\section{SECTION 3}

The differential diagnosis consideration included paroxysmal episodes affecting the occipital and parietal cortex in a "wave-like" pattern. Migraine with aura and focal seizures were considered in the differential diagnosis.

A routine sleep-deprived EEG was obtained and the results were normal. Due to the focal nature of the patient's prodromal symptoms, a brain MRI was obtained and revealed a lesion in the left occipito-parietal cortex and subcortical white matter with no associated edema or mass effect. Additionally, there were small foci of calcifications and heterogenous contrast enhancement (figure 1). Magnetic resonance angiography results were normal. 

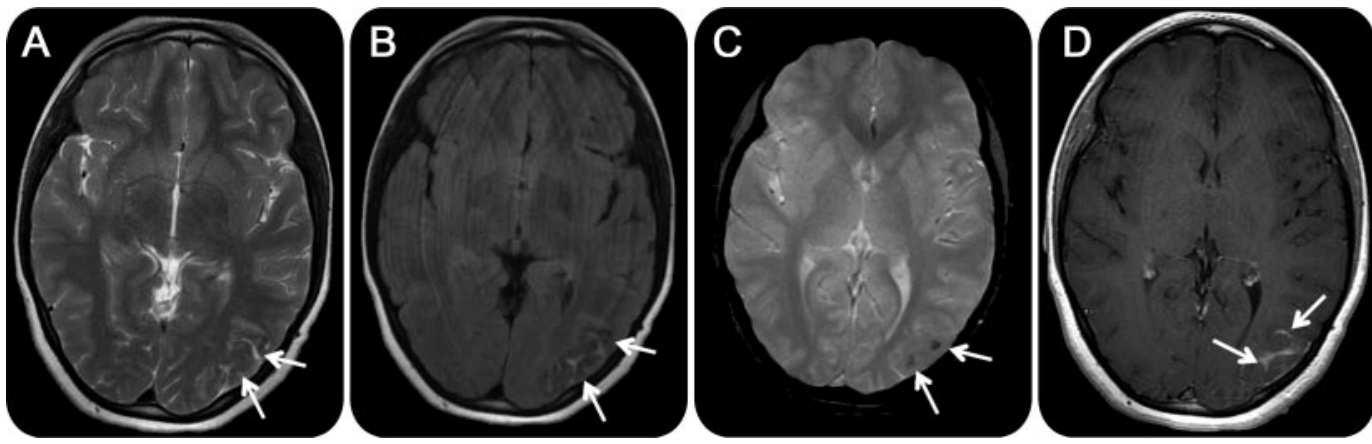

(A) Axial T2-weighted imaging shows slight signal intensity abnormality in the left occipito-parietal cortex and sulci (arrows). No peripheral edema or significant mass effect is present. (B) Axial fluid-attenuated inversion recovery image shows abnormal low signal intensity in the left occipito-parietal cortex and high signal intensity in the sulci (arrows). (C) Axial T2* image shows cortical low signal intensity foci suggestive of calcifications (arrows). (D) Axial postcontrast T1-weighted imaging after gadolinium shows enhancement in the sulci (arrows).

\section{Questions for consideration:}

1. What treatment should be considered for her complaints?
2. What differential diagnosis would you consider based on the radiologic findings and what management would you suggest at this point?

\section{SECTION 4}

Although the EEG results were normal, the stereotypical episodes along with the focal radiologic findings favored focal seizures as the most likely diagnosis. Accordingly, antiepileptic medication (levetiracetam $500 \mathrm{mg}$ twice a day) was started with partial response.

The MRI findings favored a benign tumor due to the lack of mass effect and presence of calcifications. Low-grade oligodendroglioma and dysembryoplastic neuroepithelial tumor (DNET) were initially considered. However, the heterogenous contrast enhancement raised concern for a more aggressive tumor. A vascular malformation was believed to be less likely due to the normal magnetic resonance angiogram findings.

Two approaches were discussed with the family. The first approach involved follow-up serial imaging to evaluate the rate of progression and consider surgery at a later time. The second approach was surgical resection with intraoperative electrocorticography. The decision was made to pursue the second approach in order to remove the lesion, obtain a tissue diagnosis, and remove the epileptiform cortex. Only partial resection of about $80 \%$ of the tumor was possible due to its vicinity to the vein of Labbé.

Preoperative electrocorticography showed frequent spike discharges around the border of the tu-

Figure 2 Electrocorticography preoperatively and postoperatively
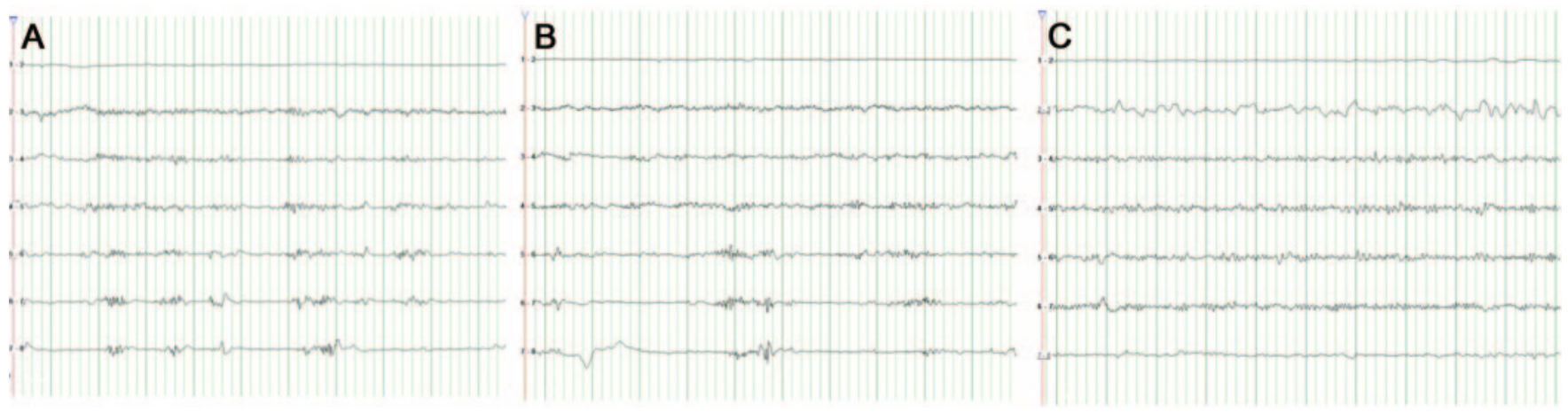

(A, B) Electrocorticography preresection shows low-amplitude spikes and polyspikes with overall amplitude suppression. (C) Electrocorticography postresection shows normal activity. 


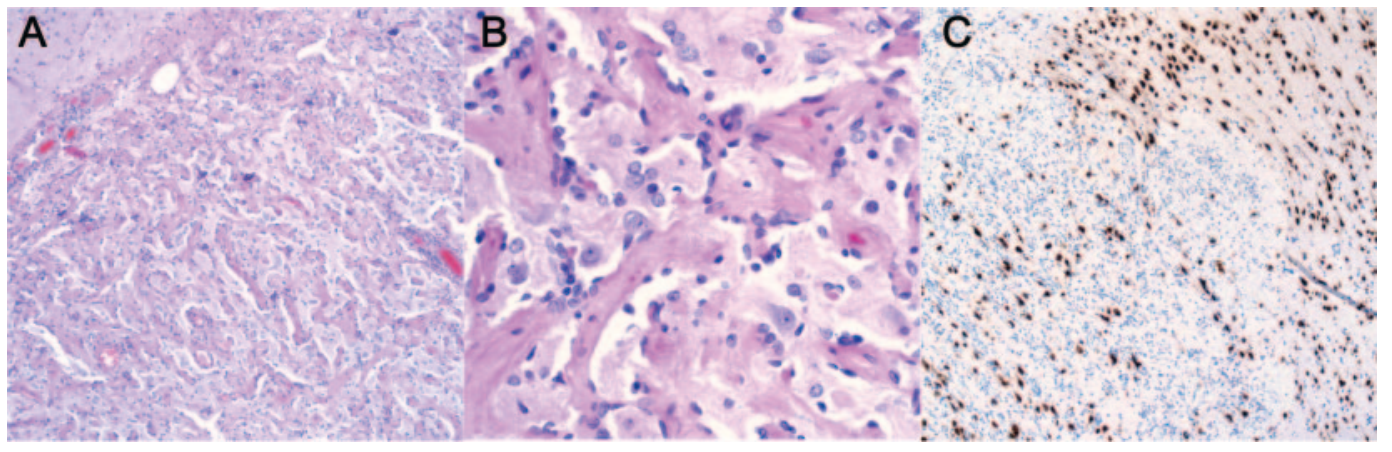

(A) Hematoxylin \& eosin stained section of cortex shows a well-demarcated cortical lesion comprised of a spindle cell proliferation extending through the full cortical thickness. Cortical laminar architecture is disrupted and abnormal neuronal clustering is prominent. (B) Hematoxylin \& eosin stained section of the cortex shows tumor cells that appear to be traveling through Virchow-Robin spaces. Note abnormally clustered entrapped neurons (arrows). (C) Cortical section stained with neuronal marker antigen shows entrapped neurons (center) next to a portion of normal cortex (right). Tumor cells did not react with synaptophysin, epithelial membrane antigen, and glial fibrillary acidic protein (not shown).

mor (figure 2). Following resection, there were persistent spikes present posteriorly but further resection was not deemed appropriate due to the tumor's proximity to the patient's visual cortex.

Tumor histology revealed meningioangiomatosis of predominant cellular type (figure 3). The levetiracetam dose was increased postoperatively due to seizure recurrence up to $1,250 \mathrm{mg}$ twice a day. The patient has remained seizure-free for 3 months. Her ophthalmologic examination results are normal.

DISCUSSION Our patient was referred to the neurology clinic with a very common complaint: a headache. It is important to consider a broad differential when evaluating patients who present with headaches so that alternative diagnoses requiring different treatment and intervention are not missed. Although our patient's symptoms could have easily fit a classic migraine diagnosis (aura lasting less than 60 minutes followed by a headache), there are important points in her symptoms that guide the clinical reasoning away from the diagnosis of migraine headaches. The International Headache Society classifies migraine headaches with certain characteristics including duration between 4 and 72 hours, associated photophobia or phonophobia, and nausea or vomiting. ${ }^{1}$ None of those characteristics were present in this case. It was the lack of typical migraine characteristics that dictated a broader differential for other paroxysmal events. Due to the stereotypic nature of the event, occipital lobe seizures followed by a postictal headache was considered a more likely diagnosis.

Migraine with aura and occipital lobe epilepsy can be difficult to distinguish but elementary visual hallucinations of occipital lobe epilepsy are fundamentally different from the aura of migraine. ${ }^{2}$ In migraines the visual aura usually starts as a flickering, uncolored, zigzag line in the center of the visual field. Symptoms gradually progress over 4-30 minutes and total duration does not exceed 60 minutes. ${ }^{2}$ In occipital seizures, the visual hallucinations often appear in the periphery of the visual field and are mainly colored and circular. They develop rapidly within seconds and are brief in duration (2-3 minutes). ${ }^{2}$ Postictal headache frequently occurs after the seizure and often generates migraine attacks. A high rate of postictal headache in occipital epilepsy was previously demonstrated. ${ }^{2}$

Although the initial EEG was negative, the presence of a structural abnormality in the brain MRI, along with the elevated clinical suspicion, prompted starting an antiepileptic medication that resulted in improvement in the patient's complaints. Routine EEG often can be normal in occipital lobe epilepsy ${ }^{2}$ but electrocorticography showed frequent focal epileptiform discharges.

The tumor biopsy revealed meningioangiomatosis, a rare, benign, hamartomatous focal lesion of leptomeninges and underlying cerebral cortex. ${ }^{3}$ Microscopically, this lesion is characterized by leptomeningeal meningothelial and meningovascular proliferation and it can be classified as mainly cellular or vascular. ${ }^{3,4} \mathrm{Cal}-$ cification, gliosis, periventricular connective tissue proliferation, dysplastic neurons, white matter cysts, and large-vessel hyalinization have also been described. ${ }^{3,4}$ Immunostaining has limited diagnostic value. ${ }^{3}$ Associated adjacent abnormalities including meningioma ${ }^{5}$ and oligodendroglioma ${ }^{6}$ have been reported.

Meningioangiomatosis is most commonly a sporadic disorder and characteristically presents with refractory localization-related epilepsy but can also present with headache or facial pain, lower cranial nerve palsies, or even be asymptomatic. ${ }^{3}$ Meningio- 
angiomatosis can also be associated with neurofibromatosis type $2,^{7}$ and these patients are usually asymptomatic and have multiple lesions. Lesions in sporadic cases are usually solitary; however, multifocal lesions have also been described. Most lesions are cortical and involve most frequently the frontal and temporal lobes. ${ }^{3}$ Extracortical lesions are more common in patients with neurofibromatosis and can include the third ventricle, cerebral peduncle, pulvinar, corpus callosum, trigeminal ganglia, and medulla. ${ }^{3}$

Some studies suggest that meningioangiomatosis has predilection for male gender ${ }^{3}$ but this is not consistent in other studies. ${ }^{8}$ The mean age of presentation is 21 years for patients without neurofibromatosis and 28 years for patients with associated neurofibromatosis. ${ }^{3}$ The treatment for meningioangiomatosis is surgical resection and it is curative if a total resection is achieved. $^{8}$

Meningioangiomatosis represents about 3\% of brain tumors associated with medically intractable epilepsy. ${ }^{9}$ In a study of 7 patients with meningioangiomatosis and seizures who underwent tumor resection, seizure-free frequency was seen in $43 \%$, improvement in seizure frequency was seen in $30 \%$, and no improvement in $28 \%$. Review of the literature by the same group showed seizure-free rates of $68 \%$, improvement in seizure frequency in 30\%, and no improvement in 5\%. In both groups, the majority of patients continued to require antiepileptic medications (71\% and $79 \%)$. $^{3}$

Radiologic characteristics suggestive of meningioangiomatosis include well-demarcated focal lesions that are isointense or hypointense in T1weighted images, commonly surrounded by an area of increased intensity on T2-weighted images, presence of calcifications, and nonhomogenous contrast enhancement. ${ }^{3,8}$

A presurgical diagnosis remains difficult because the diagnostic tools lack specificity.

MRI findings in meningioangiomatosis may mimic other pathologic processes including meningioma, oligodendroglioma, DNET, and calcified arterial-venous malformation. The need for a correct diagnosis is highlighted because of its benign course and nonrecurrent nature.

This case emphasizes the need to follow the clinical criteria to diagnose migraine headaches to avoid misdiagnosing cases where alternative diagnosis, such as occipital lobe seizures, should be considered.

\section{AUTHOR CONTRIBUTIONS}

Dr. Osorio: literature search, manuscript writing, and manuscript review. Dr. Bhatia: literature search and manuscript review. Dr. Zuccoli: radiology information and manuscript review. Dr. Holder: manuscript review.

\section{DISCLOSURE}

Dr. Osorio, Dr. Bhatia, and Dr. Zuccoli report no disclosures. Dr. Holder serves on the speakers' bureaus for Cyberonics, Inc.

\section{REFERENCES}

1. IHS Classification ICHD-II. Available at: http://www.ihsclassification.org/en/. Accessed February 6, 2011.

2. Panayiotopoulos CP. Visual phenomena and headache in occipital epilepsy: a review, a systematic study and differentiation from migraine. Epileptic Disord 1999;1:205216.

3. Wiebe S, Munoz DG, Smith S, Lee DH. Meningioangiomatosis: a comprehensive analysis of clinical and laboratory features. Brain 1999;122:709-726.

4. Savargaonkar P, Bhuiya T, Valderrama E, Farmer P. Scrape cytology of meningioangiomatosis: a report of two cases with diagnostic cytologic features. Acta Cytol 2001; 45:1069-1072.

5. Chen YY, Tiang XY, Li Z, Luo BN, Huang Q. Sporadic meningioangiomatosis-associated atypical meningioma mimicking parenchymal invasion of brain: a case report and review of the literature. Diagn Pathol 2010;5:39.

6. Lopez JI, Ereno C, Oleaga L, Areitio E. Meningioangiomatosis and oligodendroglioma in a 15-year-old boy. Arch Pathol Lab Med 1996;120:587-590.

7. Omeis I, Hillard VH, Braun A, Benzil DL, Murali R, Harter $\mathrm{DH}$. Meningioangiomatosis associated with neurofibromatosis: report of 2 cases in a single family and review of the literature. Surg Neurol 2006;65:595-603.

8. Arcos A, Serramito R, Santín JM, et al. Meningioangiomatosis: clinical-radiological features and surgical outcome. Neurocirugia 2010;21:461-466.

9. Prayson RA. Tumours arising in the setting of paediatric chronic epilepsy. Pathology 2010;42:426-431. 


\title{
Neurology
}

\author{
Clinical Reasoning: Unusual headaches \\ Maria Joana Osorio, Poonam Bhatia, Giulio Zuccoli, et al. \\ Neurology 2011;77;1649-1652 \\ DOI 10.1212/WNL.0b013e3182343286
}

This information is current as of October 24, 2011

\section{Updated Information \& Services}

\section{References}

Subspecialty Collections

Permissions \& Licensing

Reprints including high resolution figures, can be found at: http://n.neurology.org/content/77/17/1649.full

This article cites 8 articles, 0 of which you can access for free at: http://n.neurology.org/content/77/17/1649.full\#ref-list-1

This article, along with others on similar topics, appears in the following collection(s):

Cortical localization

http://n.neurology.org/cgi/collection/cortical_localization Epilepsy semiology

http://n.neurology.org/cgi/collection/epilepsy_semiology

Epilepsy surgery

http://n.neurology.org/cgi/collection/epilepsy_surgery_

Migraine

http://n.neurology.org/cgi/collection/migraine

Information about reproducing this article in parts (figures,tables) or in its entirety can be found online at:

http://www.neurology.org/about/about_the_journal\#permissions

Information about ordering reprints can be found online:

http://n.neurology.org/subscribers/advertise

Neurology ${ }^{\circledR}$ is the official journal of the American Academy of Neurology. Published continuously since 1951, it is now a weekly with 48 issues per year. Copyright Copyright (? 2011 by AAN Enterprises, Inc.. All rights reserved. Print ISSN: 0028-3878. Online ISSN: 1526-632X.

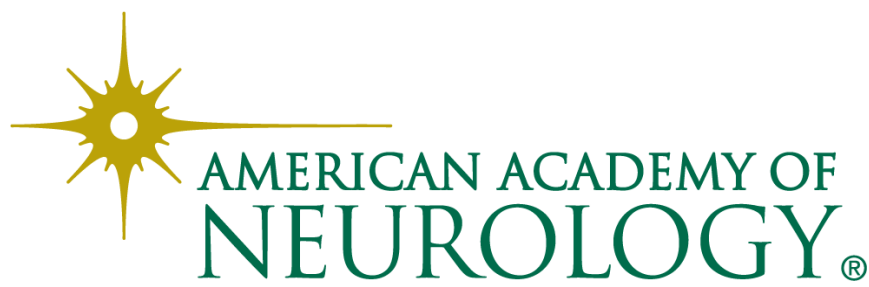

